\title{
One-Shot Coherence Distillation
}

\author{
Bartosz Regula, ${ }^{1, *}$ Kun Fang, ${ }^{2, \dagger}$ Xin Wang, ${ }^{2, \ddagger}$ and Gerardo Adesso ${ }^{1, \S}$ \\ ${ }^{1}$ School of Mathematical Sciences and Centre for the Mathematics and Theoretical Physics of Quantum Non-Equilibrium Systems, \\ University of Nottingham, University Park, Nottingham NG7 2RD, United Kingdom \\ ${ }^{2}$ Centre for Quantum Software and Information, School of Software, Faculty of Engineering and Information Technology, \\ University of Technology Sydney, New South Wales 2007, Australia
}

(Received 5 December 2017; revised manuscript received 18 April 2018; published 3 July 2018)

\begin{abstract}
We characterize the distillation of quantum coherence in the one-shot setting, that is, the conversion of general quantum states into maximally coherent states under different classes of quantum operations. We show that the maximally incoherent operations (MIO) and the dephasing-covariant incoherent operations (DIO) have the same power in the task of one-shot coherence distillation. We establish that the one-shot distillable coherence under MIO and DIO is efficiently computable with a semidefinite program, which we show to correspond to a quantum hypothesis testing problem. Further, we introduce a family of coherence monotones generalizing the robustness of coherence as well as the modified trace distance of coherence, and show that they admit an operational interpretation in characterizing the fidelity of distillation under different classes of operations. By providing an explicit formula for these quantities for pure states, we show that the one-shot distillable coherence under MIO, DIO, strictly incoherent operations, and incoherent operations is equal for all pure states.
\end{abstract}

DOI: 10.1103/PhysRevLett.121.010401

The phenomenon of quantum coherence, emerging from the fundamental property of quantum superposition, has found use in a variety of physical tasks in quantum cryptography, quantum information processing, thermodynamics, metrology, and even quantum biology [1]. Recent years have seen the development of the resource-theoretic framework of quantum coherence, establishing precise physical and mathematical laws governing the creation, manipulation, and conversion of coherence [2-5]. The archetypal example of a resource theory is quantum entanglement, and although coherence and entanglement share a large number of similarities, which allowed for many parallels and interrelations between the two resource theories to be established [5-16], the two are also very different in some aspects and can require different approaches. One particular difference is the lack of a single, physically motivated choice of free operations that best describe the allowed state manipulations in the resource theory of quantum coherence, unlike the standard choice of local operations and classical communication for entanglement [17]. It thus becomes necessary to characterize the operational properties and applications of quantum coherence under several different sets of such operations $[1,9,18-20]$, the most common ones being incoherent operations (IO) [5], strictly incoherent operations (SIO) [9], dephasing-covariant incoherent operations (DIO) $[18,19]$, and maximally incoherent operations (MIO) [2].

One of the most significant aspects of a resource theory are the rules governing state transformations under the free operations. In particular, the problem of distillation asks the following: given a canonical unit of coherence represented by the maximally coherent state $|\Psi\rangle$, what is the best rate at which we can convert copies of a state $\rho$ into copies of $|\Psi\rangle$ under a chosen set of free operations? The standard approach to this problem in quantum information theory, both in the resource theories of entanglement [21-23] and coherence $[9,24]$, is to consider the asymptotic limit - that is, assume that we have access to an unbounded number of independent and identically distributed (IID) copies of a quantum system. In a realistic setting, however, the resources are finite and the number of IID prepared states is necessarily limited. More importantly, it is very difficult to perform coherent state manipulations over large numbers of systems. Therefore, it becomes crucial to be able to characterize how well we can distill maximally coherent states from a finite number of copies of the prepared states. The study of such nonasymptotic scenarios has garnered great interest in quantum information theory [25-32], including work in the one-shot theory of entanglement distillation [33-35]. More recently, one-shot results in the resource theory of coherence [36,37] and more general quantum resource theories [38,39] were obtained.

In this Letter, we develop the framework for nonasymptotic coherence distillation, in which one has access only to a single copy of a quantum system and allows for a finite accuracy, reflecting the realistic restrictions on state transformations. In particular, we establish an exact expression for the one-shot distillable coherence under MIO and DIO, which can be efficiently computed as a semidefinite program (SDP). Interestingly, we show that the two 
quantities are in fact the same, demonstrating that $\mathrm{MIO}$ and DIO have the same power in the task of coherence distillation, and together with recent results in coherence dilution $[36,40]$ shed light on the asymptotic reversibility of state transformations under DIO. Further, we generalize two fundamental quantifiers of coherence, the robustness of coherence [41] and the modified trace distance of coherence [42], establishing a family of measures of coherence, which we show to have an operational application in characterizing the maximal fidelity of distillation under different sets of operations. We derive exact expressions for these measures for all pure states, leading to a complete characterization of pure-state one-shot distillation of coherence and showing that all the considered sets of operations - $\mathrm{IO}$, SIO, DIO, and MIO — have exactly the same power in such a task. We discuss our methods and results below and defer more technical derivations to the Supplemental Material [43].

A family of coherence monotones.-Consider a fixed orthonormal basis $\{|i\rangle\}$ in a $d$-dimensional Hilbert space $(d<\infty)$. We will use $\mathbb{D}$ to denote the set of all density matrices in this space, and for a pure state $|\psi\rangle$, we will write $\psi:=|\psi\rangle\langle\psi|$. Let $\Delta$ denote the diagonal map (fully dephasing channel) in the basis $\{|i\rangle\}$. We will denote by $\mathcal{I}$ the set of density matrices that are diagonal in this basis, i.e., $\rho \in$ $\mathbb{D}$ such that $\rho=\Delta(\rho)$, and by $\mathcal{I}^{* *}$ the cone of diagonal positive semidefinite matrices that are not necessarily normalized.

The resource theory of coherence consists of the following ingredients [5]: the set of free incoherent states, represented by $\mathcal{I}$, and the free operations, that is, a set of quantum operations that do not generate coherence. The largest possible set of such free operations are the maximally incoherent operations [2], which are given by quantum channels $\mathcal{E}$ such that $\mathcal{E}(\rho) \in \mathcal{I}$ for every $\rho \in \mathcal{I}$. The incoherent operations [5] are those for which there exists a Kraus decomposition into incoherent Kraus operators, that is, $\left\{K_{\ell}\right\}$ such that $K_{\ell} \rho K_{\ell}^{\dagger} \in \mathcal{I}^{* *}$ for all $\ell$ and all $\rho \in \mathcal{I}$. The strictly incoherent operations [9] are operations for which both $\left\{K_{\ell}\right\}$ and $\left\{K_{\ell}^{\dagger}\right\}$ are sets of incoherent operators. Finally, the dephasing-covariant incoherent operations are maps $\mathcal{E}$ such that $[\Delta, \mathcal{E}]=0$. The following strict

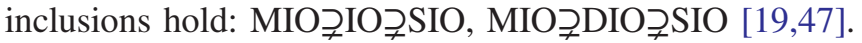

Throughout the development of the resource theory of coherence, many different quantifiers of this resource have been defined $[1,5,6,41,42,48]$. A particular example is the trace distance of coherence, given by $[5,48]$

$$
T_{\mathcal{I}}(\rho)=\min \left\{\|\rho-\sigma\|_{1} \mid \sigma \in \mathcal{I}\right\} .
$$

Although the trace distance is a fundamental quantity in quantum information theory $[49,50]$, the trace distance of coherence was found to violate the property of strong monotonicity under incoherent operations [42], which is considered as one of the requirements that a valid measure of coherence should satisfy [1,5]. Therefore, an alternative measure, called the modified trace distance of coherence, satisfying strong monotonicity under IO was proposed [42],

$$
\begin{aligned}
T_{\mathcal{I}}^{\prime}(\rho) & =\min \left\{\|\rho-\lambda \sigma\|_{1} \mid \sigma \in \mathcal{I}, \lambda \geq 0\right\} \\
& =\min \left\{\|\rho-X\|_{1} \mid X \in \mathcal{I}^{* *}\right\} .
\end{aligned}
$$

Noting that strong duality holds [51,52], we can consider the Lagrange dual of the above expression to obtain a characterization of the modified trace distance of coherence as

$$
T_{\mathcal{I}}^{\prime}(\rho)=\max \{\langle\rho, W\rangle \mid-\mathbb{1} \leq W \leq \mathbb{1}, \Delta(W) \leq 0\},
$$

with the Hilbert-Schmidt inner product $\langle X, Y\rangle=\operatorname{Tr}(X Y)$ for Hermitian operators. We then extend the above to a family of quantifiers given by SDPs of the form

$T_{\mathcal{I}}^{(m)}(\rho)=\max \{\langle\rho, W\rangle \mid-\mathbb{1} \leq W \leq m \mathbb{1}, \Delta(W) \leq 0\}$,

similar to the approach of Brandão [53] for entanglement measures. Here, we will take $m \in \mathbb{N}$, although it can be treated as a continuous parameter in general. The fact that each such measure is a valid coherence monotone can be shown by expressing $T_{\mathcal{I}}^{(m)}$ as a convex gauge function [51], and we formalize it as follows.

Proposition 1.-For each $m \geq 1, T_{\mathcal{I}}^{(m)}$ is a faithful and convex coherence measure satisfying strong monotonicity under MIO.

Note that $m=1$ gives $T_{\mathcal{I}}^{\prime}(\rho)$. For $m=d-1$, one can notice that the constraint $W \leq(d-1) \mathbb{1}$ is redundant: the other constraints ensure that the smallest eigenvalue of $W$ is at least -1 and that the trace of $W$ is at most zero, together implying that there cannot exist an eigenvalue of $W$ that is larger than $d-1$. Therefore, we get

$T_{\mathcal{I}}^{(d-1)}(\rho)=\max \{\langle\rho, W\rangle \mid-\mathbb{1} \leq W, \Delta(W) \leq 0\}=R_{\mathcal{I}}(\rho)$,

where $R_{\mathcal{I}}(\rho)$ is the robustness of coherence [41,54]. This shows that $T_{\mathcal{I}}^{(m)}$ can be thought of as a family of measures interpolating between the modified trace distance and the robustness of coherence. Notice that we clearly also have $T_{\mathcal{I}}^{(m)}(\rho)=R_{\mathcal{I}}(\rho)$ for any $m>d-1$. In general, we have that $0 \leq T_{\mathcal{I}}^{(m)}(\rho) \leq m \forall \rho \in \mathbb{D}$. It is straightforward to see by strong Lagrange duality that the family $T_{\mathcal{I}}^{(m)}$ satisfies

$$
T_{\mathcal{I}}^{(m)}(\rho)=\min _{X \in \mathcal{I}^{* *}} m \operatorname{Tr}(\rho-X)_{+}+\operatorname{Tr}(\rho-X)_{-},
$$

where $(\rho-X)_{ \pm}$denote the positive and negative parts of the Hermitian operator $\rho-X$. 
To characterize the values of the family of quantifiers $T_{\mathcal{I}}^{(m)}$ on pure states, we consider the following quantity

$$
\||\psi\rangle\left\|_{[m]}=\min _{|\psi\rangle=|x\rangle+|y\rangle}\right\||x\rangle\left\|_{\ell_{1}}+\sqrt{m}\right\||y\rangle \|_{\ell_{2}},
$$

which we (for reasons that will become clear later) call the $m$-distillation norm. We can then obtain the following result.

Theorem 1.-For any pure state $|\psi\rangle$ and any $m \geq 1$,

$$
T_{\mathcal{I}}^{(m-1)}(\psi)=\||\psi\rangle \|_{[m]}^{2}-1 .
$$

The proof of this Theorem relies on the fact that each $T_{\mathcal{I}}^{(m-1)}$ can be viewed as a robustness measure $R_{\mathcal{Q}_{m}}$ defined with respect to the set $\mathcal{Q}_{m}:=\mathcal{I} \cup(1 / m) \mathbb{D}$. Each such quantity was shown in [51] to reduce on pure states to a corresponding norm defined at the level of the underlying Hilbert space-in this case, it is precisely the $m$-distillation norm $\||\psi\rangle \|_{[m]}$.

A property of the $m$-distillation norm that will be crucial in the characterization of coherence distillation is that it can, in fact, be computed exactly. In particular, the following holds.

Theorem 2.-For a pure state $|\psi\rangle$, let $\psi_{1: k}^{\downarrow}$ denote the vector consisting of the $k$ largest (by magnitude) coefficients of $|\psi\rangle$, and analogously let $\psi_{k+1: d}^{\downarrow}$ denote the vector of the $d-k$ smallest coefficients of $|\psi\rangle$, with $\psi_{1: 0}^{\downarrow}$ being the zero vector. Then, for any pure state $|\psi\rangle$ and any integer $m \in\{1, \ldots, d\}$, we have

$$
\||\psi\rangle\left\|_{[m]}=\right\| \psi_{1: m-k^{\star}}^{\downarrow}\left\|_{\ell_{1}}+\sqrt{k^{\star}}\right\| \psi_{m-k^{\star}+1: d}^{\downarrow} \|_{\ell_{2}},
$$

where $k^{\star}=\underset{1 \leq k \leq m}{\operatorname{argmin}}\left(\left\|\psi_{m-k+1: d}^{\downarrow}\right\|_{\ell_{2}}^{2} / k\right)$.

Theorem 2 generalizes the recent result of Johnston et al. [52], where an explicit formula for the modified trace distance $T_{\mathcal{I}}^{(1)}$ was obtained for all pure states. Notice in particular that, if all coefficients of $|\psi\rangle$ satisfy $\left|\psi_{i}\right| \leq 1 / \sqrt{m}$, then $k^{\star}=m$ is optimal and we have $\||\psi\rangle \|_{[m]}=\sqrt{m}$, which means that $T_{\mathcal{I}}^{(m-1)}(\psi)$ reaches its maximum value $m-1$. As a consequence, $T_{\mathcal{I}}^{(m-1)}$ does not, in general, admit a unique maximizer in the form of the maximally coherent state. In [52], this was considered as a possible indication that this quantity is not suitable as a coherence quantifier. In the following, however, we will instead demonstrate its operational usefulness in the characterization of the fidelity of one-shot coherence distillation.

Distillation of coherence.-We will denote by $\Psi_{m}=$ $\left|\Psi_{m}\right\rangle\left\langle\Psi_{m}\right|$ the $m$-dimensional maximally coherent state $\left|\Psi_{m}\right\rangle=\sum_{i=1}^{m}(1 / \sqrt{m})|i\rangle$ in the reference basis. The distillable coherence $C_{d, \mathrm{IO}}^{\infty}(\rho)$ is the asymptotic rate at which $\Psi_{2}$ can be obtained per copy of a given state $\rho$ via incoherent operations. Winter and Yang [9] showed that the distillable coherence of an arbitrary mixed state coincides with the relative entropy of coherence $C_{r}(\rho):=\min _{\sigma \in \mathcal{I}} D(\rho \| \sigma)$ introduced in [2], where the quantum relative entropy is given as $D(\rho \| \sigma):=\operatorname{Tr} \rho(\log \rho-\log \sigma)$ with the logarithm taken in base 2. For any state $\rho$, the distillable coherence is then given by $C_{d, \mathrm{IO}}^{\infty}(\rho)=C_{r}(\rho)=S[\Delta(\rho)]-S(\rho)$.

We now consider the nonasymptotic setting. For any quantum state $\rho$, the fidelity of coherence distillation under the class of operations $\mathcal{O}$ is defined by

$$
F_{\mathcal{O}}(\rho, m):=\max _{\Lambda \in \mathcal{O}}\left\langle\Lambda(\rho), \Psi_{m}\right\rangle .
$$

The one-shot $\varepsilon$-error distillable coherence is then defined as the maximum over all distillation rates achievable under the given class of operations with an error tolerance of $\varepsilon$, that is,

$$
C_{d, \mathcal{O}}^{(1), \varepsilon}(\rho):=\log \max \left\{m \in \mathbb{N} \mid F_{\mathcal{O}}(\rho, m) \geq 1-\varepsilon\right\} .
$$

As a consequence, the asymptotic distillable coherence can be given as

$$
C_{d, \mathcal{O}}^{\infty}(\rho)=\lim _{\varepsilon \rightarrow 0} \lim _{n \rightarrow \infty} \frac{1}{n} C_{d, \mathcal{O}}^{(1), \varepsilon}\left(\rho^{\otimes n}\right) .
$$

One of the main results of this Letter is that the one-shot distillable coherence can be computed exactly, as characterized in the following result.

Theorem 3.-If $\mathcal{O} \in\{\mathrm{MIO}, \mathrm{DIO}\}$, then, for any state $\rho \in \mathbb{D}$, the fidelity of coherence distillation and one-shot $\varepsilon$ error distillable coherence can both be written as the following semidefinite programs

$$
\begin{gathered}
F_{\mathcal{O}}(\rho, m)=\max \left\{\langle G, \rho\rangle \mid 0 \leq G \leq \mathbb{1}, \Delta(G)=\frac{1}{m} \mathbb{1}\right\}, \\
C_{d, \mathcal{O}}^{(1), \varepsilon}(\rho)=\log \lfloor\max \{m \mid\langle G, \rho\rangle \geq 1-\varepsilon, \\
\left.\left.\quad 0 \leq G \leq \mathbb{1}, \Delta(G)=\frac{1}{m} \mathbb{1}\right\}\right\rfloor .
\end{gathered}
$$

The result reveals a fundamental relation between different sets of operations in the resource theory of coherence, showing that MIO and DIO have the same power in the task of coherence distillation. This correspondence is in fact surprising: not only is DIO a strict subset of MIO, it is also known that MIO is strictly more powerful than DIO in state transformations [19,47], that there exist entropic coherence monotones under DIO that are not monotones under MIO [47], and that the two sets can exhibit different operational capabilities in tasks such as coherence dilution [36]. Furthermore, since MIO constitutes the largest class of free operations in the resource theory of coherence, the result is of practical relevance, as it shows that using DIO is 
sufficient to achieve the best rates of distillation achievable under any class of free operations.

We will now show that the quantities introduced in Theorem 3 admit alternative characterizations. In particular, we will express the fidelity of distillation as a measure related to the family $T_{\mathcal{I}}^{(m)}$ introduced before and the oneshot distillable coherence as a quantum hypothesis testing problem. To do so, we will need to optimize over a larger set of matrices than the incoherent states $\mathcal{I}$, namely, the set $\mathcal{J}:=\{X \mid \operatorname{Tr}(X)=1, \Delta(X)=X\}$ of unit-trace diagonal Hermitian matrices and analogously the set $\mathcal{J}^{* *}$ of unnormalized diagonal matrices. We then define the quantities

$$
\begin{aligned}
T_{\mathcal{J}}^{(m)}(\rho) & :=\min _{X \in \mathcal{J}^{* *}} m \operatorname{Tr}(\rho-X)_{+}+\operatorname{Tr}(\rho-X)_{-} \\
& =\max \{\langle\rho, W\rangle \mid-\mathbb{1} \leq W \leq m \mathbb{1}, \Delta(W)=0\},
\end{aligned}
$$

in analogy with the measures $T_{\mathcal{I}}^{(m)}$. Following the proof of Proposition 1 , one can easily see that $T_{\mathcal{J}}^{(m)}$ are also faithful strong monotones under MIO. Let us now consider the hypothesis testing relative entropy $D_{H}^{\varepsilon}[25,55]$, defined as

$D_{H}^{\varepsilon}(\rho \| \sigma):=-\log \min \{\langle M, \sigma\rangle \mid 0 \leq M \leq \mathbb{1}, 1-\langle M, \rho\rangle \leq \varepsilon\}$.

In the setting of quantum hypothesis testing, one is interested in distinguishing between two quantum states ( $\rho$ and $\sigma$ ) by performing a test measurement $\{M, \mathbb{1}-M\}$, where $0 \leq M \leq \mathbb{1}$. The probability of incorrectly accepting state $\sigma$ as true (type-I error) is given by $\langle\mathbb{1}-M, \rho\rangle$, and the probability of incorrectly accepting state $\rho$ as true (type-II error) is given by $\langle M, \sigma\rangle$ [56]. The quantity $D_{H}^{\varepsilon}(\rho \| \sigma)$ then characterizes the minimum type-II error while constraining the type-I error to be no greater than $\varepsilon$. Alternatively, $D_{H}^{\varepsilon}(\rho \| \sigma)$ can be viewed as the operator-smoothed version of min-relative entropy [34,57]. Using this quantity, we can obtain the following result.

Proposition 2.-The fidelity of coherence distillation and the one-shot $\varepsilon$-error distillable coherence under $\mathcal{O} \in$ $\{\mathrm{MIO}, \mathrm{DIO}\}$ admit a characterization as the semidefinite programs

$$
\begin{aligned}
F_{\mathcal{O}}(\rho, m) & =\frac{1}{m}\left[T_{\mathcal{J}}^{(m-1)}(\rho)+1\right], \\
C_{d, \mathcal{O}}^{(1), \varepsilon}(\rho) & =\min _{X \in \mathcal{J}} D_{H}^{\varepsilon}(\rho \| X)-\delta,
\end{aligned}
$$

where $\delta \geq 0$ is the least number such that the solution corresponds to the logarithm of an integer.

Although the optimization in the above problems is over matrices that are not necessarily positive semidefinite, one can show that, if one of the problems admits a positive semidefinite optimal solution, then so does the other. In the particular case of $m=d$, not only does the fidelity of distillation simplify to an optimization over $\mathcal{I}$, but combining Proposition 2 with Theorem 1 of Ref. [37] we know that, in fact, $F_{\mathcal{O}}(\rho, d)$ is the same for any $\mathcal{O} \in\{\mathrm{MIO}, \mathrm{DIO}, \mathrm{SIO}, \mathrm{IO}\}$. However, the case of interest is when such a property holds for any value of $m$-we will now show that this is true for all pure states, significantly simplifying the computation of the above quantities.

We first notice that each $T_{\mathcal{I}}^{(m)}$ provides an upper bound on the corresponding $T_{\mathcal{J}}^{(m)}$, giving $F_{\mathrm{MIO}}(\rho, m) \leq$ $(1 / m)\left[T_{\mathcal{I}}^{(m-1)}(\rho)+1\right]$. To show that this bound is in fact tight for all pure states, we consider different sets of operations-SIO as well as IO. Pure-state transformations under IO and SIO are known to be fully characterized by majorization relations $[9,14,47,58]$, which allow us to lower bound the fidelity of distillation and obtain the following result.

Theorem 4.-For any pure state $|\psi\rangle$, any integer $m \geq 1$, and $\mathcal{O} \in\{\mathrm{MIO}$, DIO, SIO, IO $\}$,

$$
F_{\mathcal{O}}(\psi, m)=\frac{1}{m} \||\psi\rangle \|_{[m]}^{2} .
$$

This extends the operational equivalence between MIO and DIO in coherence distillation to the strictly smaller set SIO and has several important consequences. First, it shows that the one-shot distillable coherence of pure states under any of the classes of operations $\mathcal{O} \in\{\mathrm{MIO}, \mathrm{DIO}, \mathrm{SIO}, \mathrm{IO}\}$ is exactly the same and, in fact, can be expressed as the quantum hypothesis testing problem $C_{d, \mathcal{O}}^{(1), \varepsilon}(\psi)=\min _{\sigma \in \mathcal{I}} D_{H}^{\varepsilon}(\psi \| \sigma)-$ $\delta$ with $\delta$ as before. Second, we can use the properties of the $m$-distillation norm to obtain exact formulas for the one-shot distillable coherence. In particular, noting that $\||\psi\rangle \|_{[m]}=$ $\sqrt{m}$ [or, equivalently, $F_{\mathcal{O}}(\psi, m)=1$ ] if and only if $\||\psi\rangle \|_{\ell_{\infty}} \leq 1 / \sqrt{m}$, we see that the zero-error distillable coherence is given by

$$
C_{d, \mathcal{O}}^{(1), 0}(\psi)=\log \left\lfloor\||\psi\rangle \|_{\left.\ell_{\infty}^{-2}\right\rfloor .}\right.
$$

Relating the $m$-distillation norm with the fidelity of distillation also allows us to more easily make quantitative statements about the distillability of pure states on average. For example, in Ref. [52], it was shown that the proportion of pure states with respect to the Haar measure for which $\||\psi\rangle \|_{[2]}=1$ is given by $1-d 2^{1-d}$, which sharply tends to one as $d$ increases. In light of our results, this then shows that, with growing dimension, only an exponentially small fraction of pure states are one-shot undistillable, while a significant majority of pure states satisfy $C_{d, \mathcal{O}}^{(1), 0}(\psi) \geq 1$ and therefore allow for a zero-error one-shot distillation of at least one bit of coherence.

The results of our work have important consequences beyond the one-shot regime, in particular, for the asymptotic reversibility of state transformations in the resource 
theory of coherence - that is, the question whether the amount of coherence that can be distilled from a number of copies of a state $\rho$ (distillable coherence $C_{d}^{\infty}$ ) is the same as the amount of coherence needed to prepare the same number of copies (coherence $\operatorname{cost} C_{c}^{\infty}$ ) in the asymptotic limit of an arbitrarily large number of IID copies. It is known that the resource theory of coherence is reversible under MIO $[9,36]$, but irreversible under IO as we have $C_{d, \mathrm{IO}}^{\infty}(\rho)<C_{c, \mathrm{IO}}^{\infty}(\rho)$ in general [9]. Recently, it has been claimed that $C_{c, \mathrm{DIO}}^{\infty}(\rho)=C_{c, \mathrm{MIO}}^{\infty}(\rho)=C_{r}(\rho)$ [36], although a complete proof of this fact did not appear until [40]. Our result in Theorem 3, in particular, shows that $C_{d, \mathrm{DIO}}^{(1), \varepsilon}(\rho)=$ $C_{d, \mathrm{MIO}}^{(1), \varepsilon}(\rho)$ and therefore $C_{d, \mathrm{DIO}}^{\infty}(\rho)=C_{d, \mathrm{MIO}}^{\infty}(\rho)=C_{r}(\rho)$, complementing the claims of Ref. [36] and strengthening the asymptotic results of Ref. [40] by showing their applicability even in the one-shot case. The fact that state transformations are indeed reversible under DIO and the maximal set of operations MIO is not necessary for full reversibility contrasts with other resource theories such as entanglement, where the only set of operations known to provide asymptotic reversibility is strictly larger than the maximal set [59-62].

Conclusions. - We have characterized the operational task of one-shot coherence distillation for several classes of free operations, showing in particular that MIO and DIO have the same power in this task and providing computable expressions for the rates of distillation in terms of a quantum hypothesis testing problem. Further, we have introduced a family of coherence measures and related it to the achievable fidelity of distillation. By quantifying the introduced measures exactly on pure states and showing that they reduce to a class of much simpler vector norms, we have obtained a full characterization of one-shot coherence distillation from pure states and established that in this case all relevant sets of operations are equally useful.

Our Letter unveils several new features of the resource theory of coherence and contributes to a better understanding of the properties of the different sets of free operations, as well as generalizes and provides an operational interpretation to several coherence monotones. This yields further insight on how quantum coherence can be created and transformed in the realistic setting of finitely many quantum states available.

The possible applications of one-shot coherence distillation are multifold. Notably, the framework presented herein can be used to precisely characterize the experimentally feasible rates at which maximally coherent states, often employed as "currency" in operational tasks, can be prepared. One such application is randomness extraction [63], which can be implemented by distilling the coherence of a quantum state followed by a measurement generating uniformly random bits. Another promising way of utilizing one-shot coherence distillation is to enable coherent state preparation for direct use in quantum key distribution and quantum algorithms [64-66]. Furthermore, the comparison of the operational capabilities of different classes of operations provides, in particular, new insight about the relatively unexplored class DIO, whose relation with the so-called thermal operations could find use in the resource theory of quantum thermodynamics $[67,68]$.

We are grateful to Eric Chitambar, Min-Hsiu Hsieh, Nathaniel Johnston, Ludovico Lami, Andreas Winter, and Wei Xie for discussions. B. R. and G. A. acknowledge financial support from the European Research Council (ERC) under the Starting Grant GQCOP (Grant No. 637352). K. F. and X. W. were partly supported by the Australian Research Council (Grants No. DP120103776 and No. FT120100449).

Note added.-Recently, we became aware of an independent work by E. Chitambar [40], where the author considers the asymptotic properties of state transformations under DIO and in particular obtains a different proof that the asymptotic rate of coherence distillation under MIO and DIO is the same.

*bartosz.regula@gmail.com

†un.fang-1@ @student.uts.edu.au

xin.wang-8@student.uts.edu.au

§gerardo.adesso@nottingham.ac.uk

[1] A. Streltsov, G. Adesso, and M. B. Plenio, Rev. Mod. Phys. 89, 041003 (2017).

[2] J. Aberg, arXiv:quant-ph/0612146.

[3] G. Gour and R. W. Spekkens, New J. Phys. 10, 033023 (2008).

[4] F. Levi and F. Mintert, New J. Phys. 16, 033007 (2014).

[5] T. Baumgratz, M. Cramer, and M. B. Plenio, Phys. Rev. Lett. 113, 140401 (2014).

[6] A. Streltsov, U. Singh, H. S. Dhar, M. N. Bera, and G. Adesso, Phys. Rev. Lett. 115, 020403 (2015).

[7] J. Sperling and W. Vogel, Phys. Scr. 90, 074024 (2015).

[8] W. Vogel and J. Sperling, Phys. Rev. A 89, 052302 (2014).

[9] A. Winter and D. Yang, Phys. Rev. Lett. 116, 120404 (2016).

[10] N. Killoran, F. E. S. Steinhoff, and M. B. Plenio, Phys. Rev. Lett. 116, 080402 (2016).

[11] A. Streltsov, E. Chitambar, S. Rana, M. N. Bera, A. Winter, and M. Lewenstein, Phys. Rev. Lett. 116, 240405 (2016).

[12] S. Rana, P. Parashar, A. Winter, and M. Lewenstein, Phys. Rev. A 96, 052336 (2017).

[13] E. Chitambar and M.-H. Hsieh, Phys. Rev. Lett. 117, 020402 (2016).

[14] H. Zhu, M. Hayashi, and L. Chen, Phys. Rev. A 97, 022342 (2018).

[15] H. Zhu, Z. Ma, Z. Cao, S.-M. Fei, and V. Vedral, Phys. Rev. A 96, 032316 (2017).

[16] B. Regula, M. Piani, M. Cianciaruso, T. R. Bromley, A. Streltsov, and G. Adesso, New J. Phys. 20, 033012 (2018).

[17] R. Horodecki, P. Horodecki, M. Horodecki, and K. Horodecki, Rev. Mod. Phys. 81, 865 (2009). 
[18] E. Chitambar and G. Gour, Phys. Rev. Lett. 117, 030401 (2016).

[19] I. Marvian and R. W. Spekkens, Phys. Rev. A 94, 052324 (2016).

[20] J. I. de Vicente and A. Streltsov, J. Phys. A 50, 045301 (2017).

[21] C. H. Bennett, H. J. Bernstein, S. Popescu, and B. Schumacher, Phys. Rev. A 53, 2046 (1996).

[22] C. H. Bennett, G. Brassard, S. Popescu, B. Schumacher, J. A. Smolin, and W. K. Wootters, Phys. Rev. Lett. 76, 722 (1996).

[23] E. M. Rains, Phys. Rev. A 60, 173 (1999).

[24] E. Chitambar, A. Streltsov, S. Rana, M. N. Bera, G. Adesso, and M. Lewenstein, Phys. Rev. Lett. 116, 070402 (2016).

[25] L. Wang and R. Renner, Phys. Rev. Lett. 108, 200501 (2012).

[26] J. M. Renes and R. Renner, IEEE Trans. Inf. Theory 57, 7377 (2011).

[27] M. Tomamichel and M. Hayashi, IEEE Trans. Inf. Theory 59, 7693 (2013).

[28] M. Berta, M. Christandl, and R. Renner, Commun. Math. Phys. 306, 579 (2011).

[29] D. Leung and W. Matthews, IEEE Trans. Inf. Theory 61, 4486 (2015).

[30] N. Datta and M. H. Hsieh, IEEE Trans. Inf. Theory 59, 1929 (2013).

[31] X. Wang, W. Xie, and R. Duan, IEEE Trans. Inf. Theory 64, 640 (2017).

[32] K. Fang, X. Wang, M. Tomamichel, and R. Duan, arXiv:1706.06221.

[33] F. G. S. L. Brandão and N. Datta, IEEE Trans. Inf. Theory 57, 1754 (2011).

[34] F. Buscemi and N. Datta, J. Math. Phys. (N.Y.) 51, 102201 (2010).

[35] F. Buscemi and N. Datta, IEEE Trans. Inf. Theory 59, 1940 (2013).

[36] Q. Zhao, Y. Liu, X. Yuan, E. Chitambar, and X. Ma, Phys. Rev. Lett. 120, 070403 (2018).

[37] K. Bu, U. Singh, S.-M. Fei, A. K. Pati, and J. Wu, Phys. Rev. Lett. 119, 150405 (2017).

[38] G. Gour, Phys. Rev. A 95, 062314 (2017).

[39] G. Gour, D. Jennings, F. Buscemi, R. Duan, and I. Marvian, arXiv: 1708.04302.

[40] E. Chitambar, Phys. Rev. A 97, 050301 (2018).

[41] C. Napoli, T. R. Bromley, M. Cianciaruso, M. Piani, N. Johnston, and G. Adesso, Phys. Rev. Lett. 116, 150502 (2016).
[42] X.-D. Yu, D.-J. Zhang, G. F. Xu, and D. M. Tong, Phys. Rev. A 94, 060302 (2016).

[43] See the Supplemental Material at http://link.aps.org/ supplemental/10.1103/PhysRevLett.121.010401 for technical derivations and proofs of the results in the Letter, which includes Refs. [44-46].

[44] R. T. Rockafellar, Convex Analysis (Princeton University Press, Princeton, 1970).

[45] G. Vidal, D. Jonathan, and M. A. Nielsen, Phys. Rev. A 62, 012304 (2000).

[46] M. Sion, Pac. J. Math. 8, 171 (1958).

[47] E. Chitambar and G. Gour, Phys. Rev. A 94, 052336 (2016).

[48] S. Rana, P. Parashar, and M. Lewenstein, Phys. Rev. A 93, 012110 (2016).

[49] M. A. Nielsen and I. L. Chuang, Quantum Computation and Quantum Information, 10th ed. (Cambridge University Press, Cambridge, England, 2011).

[50] M. M. Wilde, Quantum Information Theory, 2nd ed. (Cambridge University Press, Cambridge, England, 2017).

[51] B. Regula, J. Phys. A 51, 045303 (2018).

[52] N. Johnston, C.-K. Li, and S. Plosker, arXiv:1708.06967.

[53] F. G. S. L. Brandão, Phys. Rev. A 72, 022310 (2005).

[54] M. Piani, M. Cianciaruso, T. R. Bromley, C. Napoli, N. Johnston, and G. Adesso, Phys. Rev. A 93, 042107 (2016).

[55] M. Tomamichel and M. Hayashi, IEEE Trans. Inf. Theory 59, 7693 (2013).

[56] M. Hayashi, Quantum Information Theory: Mathematical Foundation (Springer, New York, 2016).

[57] N. Datta, IEEE Trans. Inf. Theory 55, 2816 (2009).

[58] M. A. Nielsen, Phys. Rev. Lett. 83, 436 (1999).

[59] G. Vidal and J. I. Cirac, Phys. Rev. Lett. 86, 5803 (2001).

[60] G. Vidal, W. Dür, and J. I. Cirac, Phys. Rev. Lett. 89, 027901 (2002).

[61] F. G. S. L. Brandão and M. B. Plenio, Nat. Phys. 4, 873 (2008).

[62] X. Wang and R. Duan, Phys. Rev. Lett. 119, 180506 (2017).

[63] X. Yuan, H. Zhou, Z. Cao, and X. Ma, Phys. Rev. A 92, 022124 (2015).

[64] V. Scarani, H. Bechmann-Pasquinucci, N. J. Cerf, M. Dušek, N. Lütkenhaus, and M. Peev, Rev. Mod. Phys. 81, 1301 (2009).

[65] P. J. Coles, E. M. Metodiev, and N. Lütkenhaus, Nat. Commun. 7, 11712 (2016).

[66] M. Hillery, Phys. Rev. A 93, 012111 (2016).

[67] M. Lostaglio, D. Jennings, and T. Rudolph, Nat. Commun. 6, 6383 (2015).

[68] P. Ćwikliński, M. Studziński, M. Horodecki, and J. Oppenheim, Phys. Rev. Lett. 115, 210403 (2015). 